\title{
Generalized Fibonacci-Lucas Sequence
}

\author{
Bijendra Singh ${ }^{1}$, Omprakash Sikhwal, ${ }^{2, *}$, Yogesh Kumar Gupta ${ }^{3}$ \\ ${ }^{1}$ School of Studies in Mathematics, Vikram University, Ujjain-456010 (M. P.), India \\ ${ }^{2}$ Department of Mathematics, Mandsaur Institute of Technology, Mandsaur (M. P.), India \\ ${ }^{3}$ School of Studies in Mathematics, Vikram University, Ujjain, (M. P.), India \\ *Corresponding author: opbhsikhwal@rediffmail.com
}

Received September 15, 2014; Revised October 24, 2014; Accepted November 23, 2014

\begin{abstract}
The Fibonacci sequence is a source of many nice and interesting identities. A similar interpretation exists for Lucas sequence. The Fibonacci sequence, Lucas numbers and their generalization have many interesting properties and applications to almost every field. Fibonacci sequence is defined by the recurrence formula $\mathrm{F}_{\mathrm{n}}=\mathrm{F}_{\mathrm{n}-1}+\mathrm{F}_{\mathrm{n}-2}, \mathrm{n} \geq 2$ and $\mathrm{F}_{0}=0, \mathrm{~F}_{1}=1$, where $\mathrm{F}_{\mathrm{n}}$ is a $\mathrm{n}^{\text {th }}$ number of sequence. The Lucas Sequence is defined by the recurrence formula $\mathrm{L}_{\mathrm{n}}=\mathrm{L}_{\mathrm{n}-1}+\mathrm{L}_{\mathrm{n}-2}, \mathrm{n} \geq 2$ and $\mathrm{L}_{0}=2, \mathrm{~L}_{1}=1$, where $\mathrm{L}_{\mathrm{n}}$ is a $\mathrm{n}^{\text {th }}$ number of sequence. In this paper, Generalized Fibonacci-Lucas sequence is introduced and defined by the recurrence relation $B_{n}=B_{n-1}+B_{n-2}, n \geq 2$ with $\mathrm{B}_{0}=2 \mathrm{~b}, \mathrm{~B}_{1}=\mathrm{s}$, where $\mathrm{b}$ and $\mathrm{s}$ are integers. We present some standard identities and determinant identities of generalized Fibonacci-Lucas sequences by Binet's formula and other simple methods.
\end{abstract}

Keywords: Fibonacci sequence, Lucas sequence, Generalized Fibonacci-Lucas sequence, Binet's formula

Cite This Article: Bijendra Singh, Omprakash Sikhwal, and Yogesh Kumar Gupta, "Generalized FibonacciLucas Sequence." Turkish Journal of Analysis and Number Theory, vol. 2, no. 6 (2014): 193-197. doi: 10.12691/tjant-2-6-1.

\section{Introduction}

Fibonacci numbers $\mathrm{Fn}$ and Lucas numbers $\mathrm{L}_{\mathrm{n}}$ have delighted mathematicians and amateurs alike for centuries with their beauty and their propensity to pop up in quite unexpected places [3], [12] and [13]. It is well known that generalized Fibonacci and Lucas numbers play an important role in many subjects such as algebra, geometry, and number theory. Their various elegant properties and wide applications have been studied by many authors.

The Fibonacci and Lucas sequences are examples of second order recursive sequences. The Fibonacci sequence [4] is defined by the recurrence relation:

$$
F_{n}=F_{n-1}+F_{n-2}, n \geq 2 \text {, with } F_{0}=0, F_{1}=1 .
$$

The similar interpretation also exists for Lucas sequence. Lucas sequence [4] is defined by the recurrence relation:

$$
L_{n}=L_{n-1}+L_{n-2}, n \geq 2 \text { with } L_{0}=2, L_{1}=1 .
$$

Authors [1,2,3,4] and [6-13] have been generalized second order recurrence sequences by preserving the recurrence relation and altering the first two terms of the sequence, while others have generalized these sequences by preserving the first two terms of sequence but altering the recurrence relation slightly.

Horadam [1] introduced and studied properties of a generalized Fibonacci sequence $\left\{H_{n}\right\}$ and defined generalized Fibonacci sequence $\left\{H_{n}\right\}$ by the recurrence relation:

$$
H_{n+2}=H_{n+1}+H_{n}, H_{0}=q \text { and } H_{1}=p, n \geq 0,
$$

where $p, q$ are arbitrary integers.

Horadam [2] introduced and studied properties of another generalized Fibonacci sequence $\left\{w_{n}\right\}$ and defined generalized Fibonacci sequence $\left\{w_{n}\right\}$ by the recurrence relation:

$$
\begin{aligned}
& \left\{w_{n}\right\}=\left\{w_{n}(a, b ; p, q)\right\}: \mathrm{w}_{0}=a, \mathrm{w}_{1}=b, \\
& \mathrm{w}_{n}=p w_{n-1}-q w_{n-2}, \mathrm{n} \geq 2,
\end{aligned}
$$

where $a, b, p$ and $q$ are arbitrary integers.

Waddill and Sacks [10] extended the Fibonacci numbers recurrence relation and defined the sequence $\left\{P_{n}\right\}$ by recurrence relation:

$$
P_{n}=P_{n-1}+P_{n-2}+P_{n-3}, \quad n \geq 3,
$$

where $P_{0}, P_{1}$ and $P_{2}$ are not all zero given arbitrary algebraic integers.

Jaiswal [5] introduced and studied properties of generalized Fibonacci sequence $\left\{T_{n}\right\}$ and defined it by

$$
T_{n+1}=T_{n}+T_{n-1}, T_{1}=a \text { and } T_{2}=b, n \geq 1 .
$$

Falcon and Plaza [11] introduced $k^{\text {th }}$ Fibonacci sequence $\left\{F_{k, n}\right\}_{n \in N}$ and studied its properties. For any positive integer $k \geq 1, k^{\text {th }}$ Fibonacci sequence is defined by 
$F_{k, 0}=0, F_{k, 1}=1$ and $F_{k, \mathrm{n}+1}=k F_{k, \mathrm{n}}+F_{k, \mathrm{n}-1}, n \geq 1$. (1.7)

In this paper we present Generalized Fibonacci-Lucas sequence and some specific identities and some determinant identities.

\section{Generalized Fibonacci-Lucas Sequence}

Generalized Fibonacci-Lucas sequence $\left\{B_{n}\right\}_{n=0}^{\infty}$ is introduced and defined by recurrence relation:

$$
B_{n}=B_{n-1}+B_{n-2}, n \geq 2 \text { with } B_{0}=2 b \text { and } B_{1}=s,(2.1)
$$

where $b$ and $s$ are non negative integers.

The first few terms are as follows:

$$
\begin{aligned}
& B_{0}=2 b, \\
& B_{1}=s, \\
& B_{2}=2 b+s, \\
& B_{3}=2 b+2 s, \\
& B_{4}=4 b+3 s, \\
& B_{5}=6 b+5 s, \\
& B_{6}=10 b+8 s, \\
& B_{7}=16 b+13 s \text { and so on. }
\end{aligned}
$$

The characteristic equation of recurrence relation (2.1) is $t^{2}-t-1=0$. which has two real roots

$$
\alpha=\frac{1+\sqrt{5}}{2} \text { and } \beta=\frac{1-\sqrt{5}}{2} \text {. }
$$

Also, $\alpha \beta=-1, \alpha+\beta=1, \alpha-\beta=\sqrt{5}, \alpha^{2}+\beta^{2}=3$.

Generating function of generalized Fibonacci-Lucas sequence is

$$
\sum_{n=0}^{\infty} B_{n} t^{n}=B(t)=\frac{2 b+(s-2 b) t}{1-t-t^{2}} .
$$

Binet's formula of Generalized Fibonacci-Lucas sequence is defined by

$$
B_{n}=C_{1} \alpha^{n}+C_{2} \beta^{n}=C_{1}\left(\frac{1+\sqrt{5}}{2}\right)^{n}+C_{2}\left(\frac{1-\sqrt{5}}{2}\right)^{n}
$$

Here, $C_{1}=\frac{s-2 b \beta}{\sqrt{5}}$ and $C_{2}=\frac{2 b \alpha-s}{\sqrt{5}}$.

Also, $\quad C_{1} C_{2}=\frac{s^{2}-2 b s-4 b^{2}}{(\alpha-\beta)^{2}}, C_{1} \beta+C_{2} \alpha=-s+2 b$ and $C_{1}+C_{2}=2 b$.

Generalized Fibonacci-Lucas Sequence generates many classical sequences on the basis of value of $b$ and $s$.

\section{Identities of Generalized Fibonacci- Lucas Sequence}

Now some identities of generalized Fibonacci-Lucas sequence are present using generating function and Binet's formula. Authors [6,7] have been described such type identities.
Theorem (3.1). (Explicit Sum Formula) Let $G_{n}$ be the $n^{\text {th }}$ term of generalized Fibonacci-Lucas sequence. Then

$$
\left.B_{n}=2 b \sum_{k=0}^{\left[\frac{n}{2}\right.}\right]\left(\begin{array}{c}
n-k \\
\mathrm{k}
\end{array}\right)+(s-2 b) \sum_{k=0}^{\left[\frac{n-1}{2}\right]}\left(\begin{array}{c}
n-k-1 \\
\mathrm{k}
\end{array}\right) \text {. }
$$

Proof. By generating function (2.3), we have

$$
\begin{aligned}
& \sum_{n=0}^{\infty} B_{n} t^{n}=B(t)=\frac{2 b+(s-2 b) t}{1-t-t^{2}} \\
& =\{2 b+(s-2 b) t\}\left(1-t-t^{2}\right)^{-1}, \\
& =\{2 b+(s-2 b) t\}\left[1-\left(t+t^{2}\right)\right]^{-1} \\
& \sum_{n=0}^{\infty} B_{n} t^{n}=\{2 b+(s-2 b) t\} \cdot \sum_{n=0}^{\infty}\left(t+t^{2}\right)^{n}, \\
& =\{2 b+(s-2 b) t\} \cdot \sum_{n=0}^{\infty} t^{n}(1+t)^{n}, \\
& =\{2 b+(s-2 b) t\} \cdot \sum_{n=0}^{\infty} t^{n} \sum_{k=0}^{n}\left(\begin{array}{l}
n \\
k
\end{array}\right) t^{k}, \\
& =\{2 b+(s-2 b) t\} \sum_{n=0}^{\infty} \sum_{k=0}^{n} \frac{\underline{\underline{n}} \underline{\underline{k}-k}}{\underline{n}-k} t^{n+k}, \\
& =\{2 b+(s-2 b) t\} \sum_{n=0}^{\infty} \sum_{k=0}^{\infty} \frac{\underline{\underline{n}+k}}{\underline{\underline{k}} \underline{\underline{n}}} t^{n+2 k} \text {, } \\
& =\{2 b+(s-2 b) t\} \sum_{n=0}^{\infty} \sum_{k=0}^{\left[\frac{n}{2}\right]} \frac{\underline{\underline{n}-k}}{\underline{k} \underline{\underline{n}-2 k}} t^{n}, \\
& =2 b \sum_{n=0}^{\infty}\left\{\sum_{k=0}^{\left[\frac{n}{2}\right]} \frac{\underline{\lfloor n-k}}{\underline{k} \underline{n-2 k}}\right\} t^{n} \\
& +(s-2 b) \sum_{n=0}^{\infty}\left\{\sum_{k=0}^{\left[\frac{\left.\frac{n}{2}\right]}{\underline{k}\lfloor n-2 k}\right.}\right\} t^{n+1},
\end{aligned}
$$

Equating the coefficient of $t^{n}$, we obtain

$$
\sum_{n=0}^{\infty} B_{n} t^{n}=2 b \sum_{k=0}^{\left[\frac{n}{2}\right]}\left(\begin{array}{c}
n-k \\
k
\end{array}\right)+(s-2 b) \sum_{k=0}^{\left[\frac{n-1}{2}\right]}\left(\begin{array}{c}
n-k-1 \\
k
\end{array}\right) .
$$

By taking different values of $b$ and $s$ in above identity, explicit formulas can be obtained for Fibonacci and Lucas sequences.

Theorem (3.2). (Sum of First $n$ terms) Sum of first $n$ terms of Generalized Fibonacci-Lucas sequence is

$$
\sum_{k=0}^{n-1} B_{k}=B_{n+1}-s
$$

Proof. Using the Binet's formula (2.4), we have 


$$
\begin{aligned}
& \sum_{k=0}^{n-1} B_{k},=\sum_{k=0}^{n-1}\left[C_{1} \alpha^{k}+C_{2} \beta^{k}\right] \\
& =C_{1}\left[\frac{1-\alpha^{n}}{1-\alpha}\right]+C_{2}\left[\frac{1-\beta^{n}}{1-\beta}\right] \\
& =\frac{\left[\begin{array}{l}
\left.\left(C_{1}+C_{2}\right)-\left(C_{1} \beta+C_{2} \alpha\right)-\left(C_{1} \alpha^{n}+C_{1} \beta^{n}\right)\right] \\
+\alpha \beta\left(C_{1} \alpha^{n-1}+C_{1} \beta^{n-1}\right)
\end{array}\right]}{1-(\alpha+\beta)+\alpha \beta} .
\end{aligned}
$$

Using subsequent results of Binet's formula, we get

$$
\sum_{k=0}^{n-1} B_{k}=B_{n}+B_{n-1}-s=B_{n+1}-s .
$$

Theorem (3.3). (Sum of First $n$ terms with odd indices): Sum of first $n$ terms (with odd indices) of Generalized Fibonacci-Lucas sequence is

$$
\sum_{k=0}^{n-1} B_{2 k+1}=B_{2 n+1}-B_{2 n-1}-2 b=B_{2 n}-2 b .
$$

Proof. Using the Binet's formula (2.4), we have

$$
\begin{aligned}
& \sum_{k=0}^{n-1} B_{2 k+1}=\sum_{k=0}^{n-1}\left[C_{1} \alpha^{2 k+1}+C_{2} \beta^{2 k+1}\right], \\
& =-C_{1} \alpha\left[\frac{1-\alpha^{2 n}}{1-\alpha^{2}}\right]+C_{2} \beta\left[\frac{1-\beta^{2 n}}{1-\beta^{2}}\right], \\
& =\frac{\left[\begin{array}{l}
\left(C_{1} \alpha^{2 n+1}+C_{2} \beta^{2 n+1}\right)-\left(C_{1} \alpha+C_{2} \beta\right) \\
+\alpha \beta\left(C_{1} \beta+C_{2} \alpha\right)-\alpha^{2} \beta^{2}\left(C_{1} \alpha^{2 n-1}+C_{1} \beta^{2 n-1}\right)
\end{array}\right]}{\alpha^{2}+\beta^{2}-\alpha^{2} \beta^{2}-1} .
\end{aligned}
$$

Using subsequent results of Binet's formula, we get

$$
\sum_{k=0}^{n-1} B_{2 k+1}=B_{2 n+1}-B_{2 n-1}-2 b=B_{2 n}-2 b .
$$

Theorem (3.4). (Sum of First $n$ terms with even indices) Sum of first $n$ terms (with even indices) of generalized Fibonacci-Lucas sequence is given by

$$
\sum_{k=0}^{n-1} B_{2 k}=B_{2 n}-B_{2 n-2}-s+2 b=B_{2 n-1}-s+2 b .
$$

Proof. Using the Binet's formula (2.4), we have

$$
\begin{aligned}
& \sum_{k=0}^{n-1} B_{2 k}=\sum_{k=0}^{n-1}\left[C_{1} \alpha^{2 k}+C_{2} \beta^{2 k}\right] \text {, } \\
& =-C_{1}\left[\frac{1-\alpha^{2 n}}{1-\alpha^{2}}\right]+C_{2}\left[\frac{1-\beta^{2 n}}{1-\beta^{2}}\right], \\
& =\frac{\left[\begin{array}{l}
\left(C_{1} \alpha^{2 n}+C_{2} \beta^{2 n}\right)-\left(C_{1}+C_{2}\right)+\left(C_{1} \beta^{2}+C_{2} \alpha^{2}\right) \\
-\alpha^{2} \beta^{2}\left(C_{1} \alpha^{2 n-2}+C_{1} \beta^{2 n-2}\right)
\end{array}\right]}{\alpha^{2}+\beta^{2}-\alpha^{2} \beta^{2}-1} .
\end{aligned}
$$

Using subsequent results of Binet's formula, we get

$$
\sum_{k=0}^{n-1} B_{2 k}=B_{2 n}-B_{2 n-2}-s+2 b=B_{2 n-1}-s+2 b .
$$

Theorem (3.5). (Catalan's Identity) Let $B_{n}$ be the $n^{\text {th }}$ term of Generalized Fibonacci-Lucas sequence. Then

$$
\begin{aligned}
& B_{n}^{2}-B_{n+r} B_{n-r} \\
& =\frac{(-1)^{n-r}}{s^{2}-2 b s-4 b^{2}}\left(s B_{r}-2 b B_{r+1}\right)^{2}, n>r \geq 1 .
\end{aligned}
$$

Proof. Using Binet's formula (2.4), we have

$$
\begin{aligned}
& B_{n}^{2}-B_{n+r} B_{n-r} \\
&=\left(C_{1} \alpha^{n}+C_{2} \beta^{n}\right)^{2} \\
&-\left(C_{1} \alpha^{n+r}+C_{2} \beta^{n+r}\right)\left(C_{1} \alpha^{n-r}+C_{2} \beta^{n-r}\right), \\
&= C_{1} C_{2}(\alpha \beta)^{n}\left(2-\alpha^{r} \beta^{-r}-\alpha^{-r} \beta^{r}\right) \\
&= C_{1} C_{2}(\alpha \beta)^{n-r}\left(2 \alpha^{r} \beta^{r}-\alpha^{2 r}-\beta^{2 r}\right) \\
&=-C_{1} C_{2}(\alpha \beta)^{n-r}\left(\alpha^{r}-\beta^{r}\right)^{2} .
\end{aligned}
$$

Using subsequent results of Binet's formula, we get

$$
B_{n}^{2}-B_{n+r} B_{n-r}=\left(s^{2}-2 b s-4 b^{2}\right)(-1)^{n-r} \frac{\left(\alpha^{r}-\beta^{r}\right)^{2}}{(\alpha-\beta)^{2}} \text {. }
$$

Since $\frac{\alpha^{r}-\beta^{r}}{\alpha-\beta}=\frac{1}{s^{2}-2 b s-4 b^{2}}\left(s B_{r}-2 b B_{r+1}\right)$, we obtain $B_{n}^{2}-B_{n+r} B_{n-r}=\frac{(-1)^{n-r}}{s^{2}-2 b s-4 b^{2}}\left(s B_{r}-2 b B_{r+1}\right)^{2}, n>r \geq 1$.

Corollary (3.5.1). (Cassini's Identity) Let $B_{n}$ be the $n^{\text {th }}$ term of Generalized Fibonacci-Lucas sequence. Then

$$
B_{n}^{2}-B_{n+1} B_{n-1}=(-1)^{n-1}\left(s^{2}-2 b s-4 b^{2}\right), n \geq 1 .
$$

Taking $r=1$ in the Catalan's identity (3.5), the required identity is obtained.

Theorem (3.6). (d'Ocagne's Identity) Let $B_{n}$ be the $n^{\text {th }}$ term of generalized Fibonacci-Lucas sequence. Then

$$
\begin{aligned}
& B_{m} B_{n+1}-B_{m+1} B_{n} \\
& =(-1)^{n}\left(s B_{m-n}-2 b B_{m-n+1}\right), \quad m>n \geq 0 .
\end{aligned}
$$

Proof. Using Binet's formula (2.4), we have

$$
\begin{aligned}
& B_{m} B_{n+1}-B_{m+1} B_{n} \\
= & \left(C_{1} \alpha^{m}+C_{2} \beta^{m}\right)\left(C_{1} \alpha^{n+1}+C_{2} \beta^{n+1}\right) \\
& -\left(C_{1} \alpha^{m+1}+C_{2} \beta^{m+1}\right)\left(C_{1} \alpha^{n}+C_{2} \beta^{n}\right) \\
= & C_{1} C_{2}\left(\alpha^{m} \beta^{n+1}+\alpha^{n+1} \beta^{m}-\alpha^{n} \beta^{m+1}-\alpha^{m+1} \beta^{n}\right) \\
= & C_{1} C_{2}(\alpha \beta)^{n}\left[\beta\left(\alpha^{m-n}-\beta^{m-n}\right)-\alpha\left(\alpha^{m-n}-\beta^{m-n}\right)\right] \\
= & -C_{1} C_{2}(\alpha \beta)^{n}(\alpha-\beta)\left(\alpha^{m-n}-\beta^{m-n}\right) .
\end{aligned}
$$

Using subsequent results of Binet's formula, we get

$$
\begin{aligned}
& B_{m} B_{n+1}-B_{m+1} B_{n} \\
& =(-1)^{n}\left(s^{2}-2 b s-4 b^{2}\right) \frac{\left(\alpha^{m-n}-\beta^{m-n}\right)}{(\alpha-\beta)} .
\end{aligned}
$$

Since $\frac{\alpha^{m-n}-\beta^{m-n}}{\alpha-\beta}=\frac{s B_{m-n}-2 b B_{m-n+1}}{\left(s^{2}-2 b s-4 b^{2}\right)}$, we obtain 
$B_{m} B_{n+1}-B_{m+1} B_{n}=(-1)^{n}\left(s B_{m-n}-2 b B_{m-n+1}\right), m>n \geq 0$.

Theorem (3.7). (Generalized Identity) Let $B_{n}$ be the $n^{\text {th }}$ term of Generalized Fibonacci-Lucas sequence. Then

$$
\begin{aligned}
& B_{m} B_{n}-B_{m-r} B_{n+r} \\
& =(-1)^{m-r}\left(s B_{r}-2 b B_{r+1}\right)\left(s B_{n-m+r}-2 b B_{n-m+r+1}\right), \\
& n>m \geq r \geq 1 .
\end{aligned}
$$

Proof. Using Binet's formula (2.4), we have

$$
\begin{aligned}
& B_{m} B_{n}-B_{m-r} B_{n+r} \\
= & \left(C_{1} \alpha^{m}+C_{2} \beta^{m}\right)\left(C_{1} \alpha^{n}+C_{2} \beta^{n}\right) \\
& -\left(C_{1} \alpha^{m-r}+C_{2} \beta^{m-r}\right)\left(C_{1} \alpha^{n+r}+C_{2} \beta^{n+r}\right) \\
= & C_{1} C_{2}\left(\alpha^{r}-\beta^{r}\right)\left[\frac{\alpha^{m} \beta^{n}}{\alpha^{r}}-\frac{\alpha^{n} \beta^{m}}{\beta^{r}}\right] \\
= & C_{1} C_{2}(-1)^{-r}\left(\alpha^{r}-\beta^{r}\right)\left(\alpha^{m} \beta^{n+r}-\alpha^{n+r} \beta^{m}\right) \\
= & C_{1} C_{2}(-1)^{-r} \alpha^{m} \beta^{m}\left(\alpha^{r}-\beta^{r}\right)\left(\beta^{n-m+r}-\alpha^{n-m+r}\right) \\
= & -C_{1} C_{2}(-1)^{m-r}\left(\alpha^{r}-\beta^{r}\right)\left(\alpha^{n-m+r}-\beta^{n-m+r}\right) .
\end{aligned}
$$

Using subsequent results of Binet's formula, we get

$$
B_{m} B_{n}-B_{m-r} B_{n+r}
$$$$
=\frac{\left(s^{2}-2 b s-4 b^{2}\right)}{(\alpha-\beta)^{2}}(-1)^{m-r}\left(\alpha^{r}-\beta^{r}\right)\left(\alpha^{n-m+r}-\beta^{n-m+r}\right) .
$$

Since $\frac{\alpha^{r}-\beta^{r}}{\alpha-\beta}=\frac{1}{s^{2}-2 b s-4 b^{2}}\left(s B_{r}-2 b B_{r+1}\right) \quad$ and

$$
\frac{\alpha^{n-m+r}-\beta^{n-m+r}}{\alpha-\beta}=\frac{s B_{n-m+r}-2 b B_{n-m+r+1}}{\left(s^{2}-2 b s-4 b^{2}\right)} \text {. }
$$

We obtain,

$$
\begin{aligned}
& B_{m} B_{n}-B_{m-r} B_{n+r} \\
& =(-1)^{m-r}\left(s B_{r}-2 b B_{r+1}\right)\left(s B_{n-m+r}-2 b B_{n-m+r+1}\right), \\
& n>m \geq r \geq 1 .
\end{aligned}
$$

The identity (3.8) provides Catalan's, Cassini's and d'Ocagne's and other identities:

(i) If $\mathrm{m}=\mathrm{n}$, the Catalan's identity (3.5) is obtained.

(ii) If $\mathrm{m}=\mathrm{n}$ and $r=1$ in identity (3.8), the Cassini's identity (5.1) is obtained.

(iii) If $\mathrm{n}=\mathrm{m}, \mathrm{m}=n+1$ and $r=1$ in identity (3.8), the d'Ocagne's identity (3.6) is obtained.

\section{Determinant Identities}

There is a long tradition of using matrices and determinants to study Fibonacci numbers. T. Koshy [10] explained two chapters on the use of matrices and determinants. In this section, some determinant identities are presented.

Theorem(4.1). For any integers $n \geq 0$, prove that

$$
\left|\begin{array}{lll}
B_{n+1} & \mathrm{~B}_{n+2} & \mathrm{~B}_{n+3} \\
B_{n+4} & \mathrm{~B}_{n+5} & \mathrm{~B}_{n+6} \\
B_{n+7} & \mathrm{~B}_{n+8} & \mathrm{~B}_{n+9}
\end{array}\right|=0 .
$$

Proof.

$$
\text { Let } \Delta=\left|\begin{array}{lll}
B_{n+1} & \mathrm{~B}_{n+2} & \mathrm{~B}_{n+3} \\
B_{n+4} & \mathrm{~B}_{n+5} & \mathrm{~B}_{n+6} \\
B_{n+7} & \mathrm{~B}_{n+8} & \mathrm{~B}_{n+9}
\end{array}\right|
$$

Applying $C_{1} \rightarrow C_{1}+C_{2}$, we get

$$
\text { Let } \Delta=\left|\begin{array}{lll}
B_{n+3} & \mathrm{~B}_{n+2} & \mathrm{~B}_{n+3} \\
B_{n+6} & \mathrm{~B}_{n+5} & \mathrm{~B}_{n+6} \\
B_{n+9} & \mathrm{~B}_{n+8} & \mathrm{~B}_{n+9}
\end{array}\right|
$$

Since two columns are identical,we obtained required result.

Theorem (4.2). For any integer $n \geq 0$, prove that

$$
\left|\begin{array}{lcc}
B_{n}-B_{n+1} & \mathrm{~B}_{n+1}-B_{n+2} & \mathrm{~B}_{n+2}-B_{n} \\
B_{n+1}-B_{n+2} & \mathrm{~B}_{n+2}-B_{n} & \mathrm{~B}_{n}-B_{n+1} \\
B_{n+2}-B_{n} & \mathrm{~B}_{n}-B_{n+1} & \mathrm{~B}_{n+1}-B_{n+2}
\end{array}\right|=0 .(4.2)
$$

Proof.

$$
\text { Let } \Delta=\left|\begin{array}{lcc}
B_{n}-B_{n+1} & \mathrm{~B}_{n+1}-B_{n+2} & \mathrm{~B}_{n+2}-B_{n} \\
B_{n+1}-B_{n+2} & \mathrm{~B}_{n+2}-B_{n} & \mathrm{~B}_{n}-B_{n+1} \\
B_{n+2}-B_{n} & \mathrm{~B}_{n}-B_{n+1} & \mathrm{~B}_{n+1}-B_{n+2}
\end{array}\right| \text {. }
$$

By applying $C_{1} \rightarrow \mathrm{C}_{1}+C_{2}+C_{3}$ and expanding along first row, we obtained required result.

Theorem (4.3). For any integer $n \geq 0$, prove that

$$
\left|\begin{array}{ccc}
1 & 1 & 1 \\
\mathrm{~B}_{n} & \mathrm{~B}_{n+1} & \mathrm{~B}_{n+2} \\
B_{n+1}+B_{n+2} & \mathrm{~B}_{n}+B_{n+2} & \mathrm{~B}_{n}+B_{n+1}
\end{array}\right|=0 .(4.3)
$$

Proof.

$$
\text { Let } \Delta=\left|\begin{array}{ccc}
1 & 1 & 1 \\
\mathrm{~B}_{n} & \mathrm{~B}_{n+1} & \mathrm{~B}_{n+2} \\
B_{n+1}+B_{n+2} & \mathrm{~B}_{n}+B_{n+2} & \mathrm{~B}_{n}+B_{n+1}
\end{array}\right| \text {. }
$$

Applying $R_{3} \rightarrow R_{3}+R_{2}$, we get

$$
\Delta=\left|\begin{array}{lcc}
1 & 1 & 1 \\
B_{n} & \mathrm{~B}_{n+1} & \mathrm{~B}_{n+2} \\
2 B_{n+2} & 2 B_{n+2} & 2 B_{n+2}
\end{array}\right| .
$$

Taking common out $2 B_{n+2}$ from third row,

$$
\Delta=2 B_{n+2}\left|\begin{array}{ccc}
1 & 1 & 1 \\
B_{n} & \mathrm{~B}_{n+1} & \mathrm{~B}_{n+2} \\
1 & 1 & 1
\end{array}\right| .
$$

Since two rows are identical, thus we obtained required result.

Theorem (4.4). For any integer $n \geq 0$, prove that

$$
\begin{aligned}
& \left|\begin{array}{ccc}
\mathrm{B}_{n} & \mathrm{~B}_{n}+B_{n+1} & \mathrm{~B}_{n}+B_{n+1}+B_{n+2} \\
2 B_{n} & 2 \mathrm{~B}_{n}+3 B_{n+1} & 2 \mathrm{~B}_{n}+3 B_{n+1}+4 B_{n+2} \\
3 \mathrm{~B}_{n} & 3 \mathrm{~B}_{n}+6 B_{n+1} & 3 \mathrm{~B}_{n}+6 B_{n+1}+12 B_{n+2}
\end{array}\right| \\
& =3 B_{n} B_{n+1} B_{n+2} .
\end{aligned}
$$


Let $\Delta=\left|\begin{array}{ccl}\mathrm{B}_{n} & \mathrm{~B}_{n}+B_{n+1} & \mathrm{~B}_{n}+B_{n+1}+B_{n+2} \\ 2 B_{n} & 2 \mathrm{~B}_{n}+3 B_{n+1} & 2 \mathrm{~B}_{n}+3 B_{n+1}+4 B_{n+2} \\ 3 \mathrm{~B}_{n} & 3 \mathrm{~B}_{n}+6 B_{n+1} & 3 \mathrm{~B}_{n}+6 B_{n+1}+12 B_{n+2}\end{array}\right|$.

Applying $R_{2} \rightarrow R_{2}-2 R_{1}, R_{3} \rightarrow R_{3}-3 R_{1}$, we get

$$
\Delta=\left|\begin{array}{ccc}
B_{n} & \mathrm{~B}_{n}+\mathrm{B}_{n+1} & \mathrm{~B}_{n}+\mathrm{B}_{n+1}+B_{n+2} \\
0 & \mathrm{~B}_{n+1} & \mathrm{~B}_{n+1}+2 \mathrm{~B}_{n+2} \\
0 & 3 \mathrm{~B}_{n+1} & 3 \mathrm{~B}_{n+1}+9 B_{n+2}
\end{array}\right| .
$$

Applying $R_{3} \rightarrow R_{3}-3 R_{2}$ and expanding along first row, we obtained required result.

Theorem (4.5). For any integer $n \geq 0$, prove that

$$
\left|\begin{array}{ccc}
0 & \mathrm{~B}_{n} B_{n+1}^{2} & \mathrm{~B}_{n} B_{n+2}^{2} \\
B_{n}^{2} B_{n+1} & 0 & \mathrm{~B}_{n+1} B_{n+2}^{2} \\
B_{n}^{2} B_{n+2} & \mathrm{~B}_{n+2} B_{n+1}^{2} & 0
\end{array}\right|=2 B_{n}^{3} B_{n+1}^{3} B_{n+2}^{3} .
$$

Proof.

$$
\text { Let } \Delta=\left|\begin{array}{ccc}
0 & \mathrm{~B}_{n} B_{n+1}^{2} & \mathrm{~B}_{n} B_{n+2}^{2} \\
B_{n}^{2} B_{n+1} & 0 & \mathrm{~B}_{n+1} B_{n+2}^{2} \\
B_{n}^{2} B_{n+2} & \mathrm{~B}_{n+2} B_{n+1}^{2} & 0
\end{array}\right| \text {. }
$$

Taking common out $B_{n}^{2}, \mathrm{~B}_{n+1}^{2}, \mathrm{~B}_{n+2}^{2}$ from $C_{1}, C_{2}, C_{3}$ respectively, we get

$$
\Delta=\mathrm{B}_{n}^{2} B_{n+1}^{2} B_{n+2}^{2}\left|\begin{array}{ccc}
0 & \mathrm{~B}_{n} & \mathrm{~B}_{n} \\
B_{n+1} & 0 & \mathrm{~B}_{n+1} \\
B_{n+2} & \mathrm{~B}_{n+2} & 0
\end{array}\right| .
$$

Taking common out $B_{n}, \mathrm{~B}_{n+1}, \mathrm{~B}_{n+2}$ from $R_{1}, \mathrm{R}_{2}, \mathrm{R}_{3}$ respectively and expanding along first row, we obtained required result.

Theorem (4.6). For any integer $n \geq 0$, prove that

$$
\left|\begin{array}{ccc}
\mathrm{B}_{\mathrm{n}} & \mathrm{F}_{\mathrm{n}} & 1 \\
\mathrm{~B}_{\mathrm{n}+1} & \mathrm{~F}_{\mathrm{n}+1} & 1 \\
\mathrm{~B}_{\mathrm{n}+2} & \mathrm{~F}_{\mathrm{n}+2} & 1
\end{array}\right|=\left[F_{n} B_{n+1}-B_{n} F_{n+1}\right]
$$

Proof: Let $\Delta=\left|\begin{array}{ccc}B_{n} & F_{n} & 1 \\ B_{n+1} & F_{n+1} & 1 \\ B_{n+2} & F_{n+2} & 1\end{array}\right|$

Assume $B_{n}=a, B_{n+1}=b, B_{n+2}=a+b$ and $F_{n}=p, F_{n+1}=q$, $\mathrm{F}_{\mathrm{n}+2}=\mathrm{p}+\mathrm{q}$.

Now substituting the above values in determinant, we get

$$
\Delta=\left|\begin{array}{ccc}
a & p & 1 \\
b & q & 1 \\
a+b & p+q & 1
\end{array}\right|
$$

Applying $R_{1} \rightarrow R_{1}-R_{2}$

$$
\Delta=\left|\begin{array}{ccc}
a-b & p-q & 0 \\
b & q & 1 \\
a+b & p+q & 1
\end{array}\right|
$$

Applying $R_{2} \rightarrow R_{2}-R_{3}$

$$
\Delta=\left|\begin{array}{ccc}
a-b & p-q & 0 \\
-a & -p & 0 \\
a+b & p+q & 1
\end{array}\right|=(p b-a q)
$$

Substituting the values of $a, b, p$ and $q$, we get required result.

Similarly following identities can be derived:

Theorem (4.8). For any integer $n \geq 0$, prove that

$$
\left|\begin{array}{ccc}
B_{n} & L_{n} & 1 \\
B_{n+1} & L_{n+1} & 1 \\
B_{n+2} & L_{n+2} & 1
\end{array}\right|=2\left(L_{n} B_{n+1}-B_{n} L_{n+1}\right)
$$

Theorem (4.9). For any integer $n \geq 0$, prove that

$$
\left|\begin{array}{ccc}
B_{n}+B_{n+1} & B_{n+1}+B_{n+2} & B_{n+2}+B_{n} \\
B_{n+2} & B_{n} & B_{n+1} \\
1 & 1 & 1
\end{array}\right|=0
$$

Theorem 4.(10). For any integer $n \geq 0$, prove that

$$
\left|\begin{array}{ccc}
1+B_{n} & B_{n+1} & B_{n+2} \\
B_{n} & 1+B_{n+1} & B_{n+2} \\
B_{n} & B_{n+1} & 1+B_{n+2}
\end{array}\right|=1+B_{n}+B_{n+1}+B_{n+2}(4.10)
$$

\section{Conclusions}

In this paper, Generalized Fibonacci-Lucas sequence is introduced. Some standard identities of generalized Fibonacci-Lucas sequence have been obtained and derived using generating function and Binet's formula. Also some determinant identities have been established and derived.

\section{References}

[1] A. F. Horadam: A Generalized Fibonacci Sequence, American Mathematical Monthly, Vol. 68. (5), 1961, 455-459.

[2] A. F. Horadam: Basic Properties of a Certain Generalized Sequence of Numbers, The Fibonacci Quarterly, Vol. 3 (3), 1965, $161-176$.

[3] A.T. Benjamin and D. Walton: Counting on Chebyshev polynomials, Math. Mag. 82, 2009, 117-126.

[4] B. Singh, O. Sikhwal and S. Bhatnagar: Fibonacci-Like Sequence and its Properties, Int. J. Contemp. Math. Sciences, Vol. 5 (18), 2010, 859-868.

[5] B. Singh, S. Bhatnagar and O. Sikhwal: Fibonacci-Like Polynomials and some Identities, International Journal of Advanced Mathematical Sciences, 1 (3), (2013) 152-157.

[6] B. Singh, S. Bhatnagar and O. Sikhwal: Fibonacci-Like Sequence, International Journal of Advanced Mathematical Sciences, 1 (3) (2013) 145-151.

[7] B. Singh, S. Bhatnagar and O. Sikhwal: Generalized Identties of Companion Fibonacci-Like Sequences, Global Journal of Mathematical Analysis, 1 (3), 2013, 104-109.

[8] D. V. Jaiswal: On a Generalized Fibonacci Sequence, Labdev J. Sci. Tech. Part A 7, 1969, 67-71.

[9] M. Edson and O. Yayenie: A New Generalization of Fibonacci sequence and Extended Binet's Formula, Integers Vol. 9, 2009, 639-654.

[10] M. E. Waddill and L. Sacks: Another Generalized Fibonacci Sequence, The Fibonacci Quarterly, Vol. 5 (3), 1967, 209-222.

[11] S. Falcon and A. Plaza: On the Fibonacci $K$ - Numbers, Chaos, Solutions \& Fractals, Vol. 32 (5), 2007, 1615-1624,

[12] S. Vajda, Fibonacci \& Lucas Numbers, and the Golden Section, Theory and Applications, Ellis Horwood Ltd., Chichester, 1989.

[13] T. Koshy, Fibonacci and Lucas Numbers with Applications, Wiley-Interscience Publication, New York (2001). 